\title{
Revisiting interval protection, a.k.a. partial cell suppression, for tabular data *
}

\author{
Jordi Castro $^{1 \star \star}$ and Anna Via ${ }^{2}$ \\ 1 Department of Statistics and Operations Research, \\ Universitat Politècnica de Catalunya, \\ Jordi Girona 1-3, 08034 Barcelona, Catalonia, \\ jordi.castro@upc.edu. \\ 2 School of Mathematics and Statistics \\ Universitat Politècnica de Catalunya, \\ Pau Gargallo 5, 08028 Barcelona, Catalonia, \\ annaa35@gmail.com.
}

\begin{abstract}
Interval protection or partial cell suppression was introduced in "M. Fischetti, J.-J. Salazar, Partial cell suppression: A new methodology for statistical disclosure control, Statistics and Computing, 13, 1321,2003 " as a "linearization" of the difficult cell suppression problem. Interval protection replaces some cells by intervals containing the original cell value, unlike in cell suppression where the values are suppressed. Although the resulting optimization problem is still huge - as in cell suppression, it is linear, thus allowing the application of efficient procedures. In this work we present preliminary results with a prototype implementation of Benders decomposition for interval protection. Although the above seminal publication about partial cell suppression applied a similar methodology, our approach differs in two aspects: (i) the boundaries of the intervals are completely independent in our implementation, whereas the one of 2003 solved a simpler variant where boundaries must satisfy a certain ratio; (ii) our prototype is applied to a set of seven general and hierarchical tables, whereas only three two-dimensional tables were solved with the implementation of 2003.
\end{abstract}

Key words: statistical disclosure control, tabular data, interval protection, cell suppression, linear optimization, large-scale optimization.

\section{Introduction}

Post-tabular data protection methods are based on modifying or suppressing some of the table cells, yet satisfying the table additivity (that is, the

\footnotetext{
* Supported by grant MTM2015-65362-R of the Spanish Ministry of Economy and Competitiveness.

** Corresponding author
} 
sum of the "inner" cells has to be equal to the marginal cell) and preserving the original value of a subset of cells (e.g., some subtotal or total cells). This is the main difference compared to pre-tabular methods, which at the same time cannot guarantee table additivity and the original value of a subset of cells. Among post-tabular data protection methods we find cell suppression [9] and controlled tabular adjustment [3], both formulating difficult mixed integer linear optimization problems. More details can be found in the monograph [12] and the survey [5].

Interval protection or partial cell suppression was introduced in [10] as a linearization of the difficult cell suppression problem. Unlike in cell suppression, interval protection replaces some cell values by intervals containing the true value. From those intervals, no attacker can be able to recompute the true value within some predefined lower and upper protection levels. One of the great advantages of interval suppression against alternative approaches is that the resulting optimization problem is convex and continuous, which means that theoretically it can be efficiently solved in polynomial time by, for instance, interior-point methods 13 . Therefore, theoretically, this approach is valid for big tables from the bigdata era.

However, attempting to solve the resulting "monolithic" linear optimization model by some state-of-the-art solver is almost impossible for huge tables: we will either exhaust the RAM memory of the computer, or we will require a large CPU time. Alternative approaches to be tried include a Benders decomposition of this huge linear optimization problem. In this work we present preliminary results with a prototype implementation of Benders decomposition. A similar approach was used in the seminal publication [10] about partial cell suppression. However, this work differs in two substantial aspects: (i) our implementation considers two independent boundaries for each cell interval, whereas those two boundaries were forced to satisfy a ratio in the code of [10] (that is, actually only one boundary was considered in the 2003 code, thus solving a simpler variant of the problem); (ii) we applied our prototype to a set of seven general and hierarchical tables, where results for only three two-dimensional tables were reported in [10]. As we will see, our "not-too efficient and tuned" classical Benders decomposition prototype still outperforms state-of-theart solvers in these complex tables.

The paper is organized as follows. Section 2 describes the general interval protection method. Section 3 outlines the Benders solution approach. The particular form of Benders for interval protection is shown in Section 4. which is illustrated by a small example in Subsection 4.1. Finally, Sec- 
tion 5 reports computational results with some general and hierarchical tables.

\section{The general interval protection problem formulation}

We are given a table (i.e., a set of cells $a_{i}, i \in \mathcal{N}=\{1, \ldots, n\}$ ), satisfying $m$ linear relations $A a=b, A \in \mathbb{R}^{m \times n}, b \in \mathbb{R}^{m}$. Any set of values $x$ satisfying $A x=b, l \leq x \leq u$, is a valid table, $l \in \mathbb{R}^{n}, u \in \mathbb{R}^{n}$ being known a priori lower and upper bounds for cell values. For positive tables we have $l_{i}=0, u_{i}=+\infty, i=1, \ldots, n$, but the procedure here outlined is also valid for general tables. For instance, we may consider the cells provide information about some attribute for several individual states (e.g., member states of European Union), as well as the highest-level of aggregated information (e.g., at European Union level). The set of multistate cells, or cells providing this highest-level of aggregated information could be the ones to be replaced by intervals, and they will be denoted as $\mathcal{H} \subseteq \mathcal{N}$.

Let $\mathcal{F}, \mathcal{S}, \mathcal{M}$ be a partition of $\mathcal{N}$, i.e., $\mathcal{N}=\mathcal{F} \cup \mathcal{S} \cup \mathcal{M}$, and $\mathcal{F} \cap \mathcal{S}=$ $\mathcal{F} \cap \mathcal{M}=\mathcal{S} \cap \mathcal{M}=\emptyset . \mathcal{S}$ is the set of sensitive cells to be protected, with upper and lower protection levels $u p l_{s}$ and $l p l_{s}$ for each cell $s \in \mathcal{S}$. $\mathcal{F}$ is the set of cells whose values are known (e.g., they have been previously published by individual states). $\mathcal{M}$ is the set of non-sensitive and non previously published cells. To simplify the formulation of the forthcoming optimization problems, we can assume that for $f \in \mathcal{F}$ we have $l_{f}=$ $u_{f}=a_{f}$, and then cells from $\mathcal{F}$ can be considered elements of $\mathcal{M}$, that is, $\mathcal{M} \leftarrow \mathcal{M} \cup \mathcal{F}$ and $\mathcal{F} \leftarrow \emptyset$. Following our example, we have that, in general, cells in $\mathcal{S}$ provide information at state level, but in some cases multi-state cells may also be sensitive; thus we may have $\mathcal{S} \cap \mathcal{H} \neq \emptyset$. In a similar way, since multi-state cells may not have been previously published we may also have $\mathcal{M} \cap \mathcal{H} \neq \emptyset$. To make the formulation more general our only assumption will be that $\mathcal{H} \subseteq \mathcal{N}$. When $\mathcal{H}=\mathcal{N}$ we just have the standard "interval protection" or "partial cell suppression" introduced in [10.

Our purpose is to publish the set of smallest intervals $\left[l b_{h}, u b_{h}\right]$-where $l_{h} \leq l b_{h}$ and $u b_{h} \leq u_{h}$ - for each cell $h \in \mathcal{H}$ instead of the real value $a_{h} \in\left[l b_{h}, u b_{h}\right]$, such that, from these intervals, no attacker can determine that $a_{s} \in\left(a_{s}-l p l_{s}, a_{s}+u p l_{s}\right)$ for all sensitive cells $s \in \mathcal{S}$. This means that

$$
\underline{a_{s}} \leq a_{s}-l p l_{s} \text { and } \overline{a_{s}} \geq a_{s}+u p l_{s},
$$

$\underline{a_{s}}$ and $\overline{a_{s}}$ being defined as 


$$
\begin{aligned}
& \underline{a_{s}}=\min x_{s} \quad \overline{a_{s}}=\max x_{s} \\
& \text { s.to } A x=b \quad \text { s.to } A x=b \\
& l_{i} \leq x_{i} \leq u_{i} \quad i \in \mathcal{N} \backslash \mathcal{H} \quad \text { and } \quad l_{i} \leq x_{i} \leq u_{i} \quad i \in \mathcal{N} \backslash \mathcal{H} \\
& l b_{i} \leq x_{i} \leq u b_{i} i \in \mathcal{H} \quad l b_{i} \leq x_{i} \leq u b_{i} i \in \mathcal{H}
\end{aligned}
$$

Clearly, for cells $i \in \mathcal{H} \cap \mathcal{S}$, (11) and (2) imply that $l b_{i} \leq a_{i}-l p l_{i}$ and $u b_{i} \geq a_{i}+u p l_{i}$.

The previous problem can be formulated as a large-scale linear optimization problem. For each primary cell $s \in \mathcal{S}$, two auxiliary vectors $x^{l, s} \in \mathbb{R}^{n}$ and $x^{u, s} \in \mathbb{R}^{n}$ are introduced to impose, respectively, the lower and upper protection requirement of (1). The problem formulation is as follows:

$$
\begin{aligned}
& \min \sum_{i \in \mathcal{H}} w_{i}\left(u b_{i}-l b_{i}\right) \\
& \text { s.to }{ }^{i \in \mathcal{H}} \quad A x^{l, s}=b \\
& l_{i} \leq x_{i}^{l, s} \leq u_{i} \quad i \in \mathcal{N} \backslash \mathcal{H} \\
& l b_{i} \leq x_{i}^{l, s} \leq u b_{i} \quad i \in \mathcal{H} \\
& x_{s}^{l, s} \leq a_{s}-l p l_{s} \quad \forall s \in \mathcal{S} \\
& \left.\begin{array}{rl}
A x^{u, s} & =b \\
l_{i} \leq x_{i}^{u, s} & \leq u_{i} \quad i \in \mathcal{N} \backslash \mathcal{H} \\
l b_{i} \leq x_{i}^{u, s} & \leq u b_{i} \quad i \in \mathcal{H} \\
x_{s}^{u, s} & \geq a_{s}+u p l_{s}
\end{array}\right\} \\
& l_{i} \leq l b_{i} \leq a_{i} \quad i \in \mathcal{H} \\
& a_{i} \leq u b_{i} \leq u_{i} \quad i \in \mathcal{H}
\end{aligned}
$$

where $w_{i}$ is a weight for the information loss associated with cell $a_{i}$.

Problem (3) is very large (easily in the order of millions of variables and constraints), but it is linear (no binary, no integer variables), and thus theoretically it can be efficiently solved in polynomial time by general or by specialized interior-point algorithms [137].

\section{Outline of Benders decomposition}

Benders decomposition [2] was suggested for problems with two types of variables, one of them considered as "complicating variables". In MILP models complicating variables are the binary/integer ones; in continuous problems, the complicating variables are usually associated to linking variables between groups of constraints (i.e., variables $l b$ and $u b$ in (3) ) . 
Consider the following primal problem $(P)$ with two groups of variables $(x, y)$

$$
\begin{array}{ll}
\min & c^{\top} x+d^{\top} y \\
\text { s. to } & A_{1} x+A_{2} y=b \\
& x \geq 0 \\
& y \in Y,
\end{array}
$$

where $y$ are the complicating variables, $c, x \in \mathbb{R}^{n_{1}}, d, y \in \mathbb{R}^{n_{2}}, A_{1} \in \mathbb{R}^{m \times n_{1}}$ and $A_{2} \in \mathbb{R}^{m \times n_{2}}$. Fixing some $y \in Y$, we obtain:

$$
\begin{aligned}
& \min c^{\top} x \\
& \text { (Q) } \quad \text { s. to } \quad A_{1} x=b-A_{2} y \\
& x \geq 0 \text {. }
\end{aligned}
$$

The dual of $(Q)$ is:

$$
\begin{array}{lll} 
& \max & u^{\top}\left(b-A_{2} y\right) \\
\left(Q_{D}\right) \quad \text { s. to } & A_{1}^{\top} u \leq c \\
& u \in \mathbb{R}^{m} .
\end{array}
$$

It is known that if $\left(Q_{D}\right)$ has a solution then $(Q)$ has a solution too, and both objective functions coincide; if $\left(Q_{D}\right)$ is unbounded, then $(Q)$ is infeasible. Let assume that $\left(Q_{D}\right)$ is never infeasible (in the interval protection problem this is always the case). If, as notation convention, we consider that the objective of $(Q)$ is $+\infty$ when it is infeasible, then $(P)$ can be written as

$$
\begin{array}{lll}
\left(P^{\prime}\right) \quad \min & \left\{d^{\top} y+\max \left\{u^{\top}\left(b-A_{2} y\right) \mid A_{1}^{\top} u \leq c, u \in \mathbb{R}^{m}\right\}\right\} \\
& \text { s. to } \quad y \in Y .
\end{array}
$$

Let $U=\left\{u \mid A_{1}^{\top} u \leq c, u \in \mathbb{R}^{m}\right\}$ be the convex feasible set of $\left(Q_{D}\right)$. By Minkowski representation we know that every point $u \in U$ may be represented as a convex combination of the vertices $u^{1}, \ldots, u^{s}$ and extreme rays $v^{1}, \ldots, v^{t}$ of the convex polytope $U$. Therefore any $u \in U$ may be written as

$$
\begin{gathered}
u=\sum_{i=1}^{s} \lambda_{i} u^{i}+\sum_{j=1}^{t} \mu_{j} v^{j} \\
\sum_{i=1}^{s} \lambda_{i}=1 \\
\lambda_{i} \geq 0 \quad i=1, \ldots, s \\
\mu_{j} \geq 0 \quad j=1, \ldots, t .
\end{gathered}
$$

If $v^{j^{\top}}\left(b-A_{2} y\right)>0$ for some $j \in\{1, \ldots, t\}$ then $\left(Q_{D}\right)$ is unbounded, and thus $(Q)$ is infeasible. We then impose

$$
v^{j^{\top}}\left(b-A_{2} y\right) \leq 0 \quad j=1, \ldots, t .
$$


The optimal solution of $\left(Q_{D}\right)$ is then known to be in a vertex of $U$, and $\left(P^{\prime}\right)$ may be rewritten as

$$
\begin{array}{lll} 
& \min \quad d^{\top} y+\max _{i=1, \ldots, s}\left(u^{i^{\top}}\left(b-A_{2} y\right)\right) \\
\left(P^{\prime \prime}\right) \quad \text { s. to } \quad & v^{j^{\top}}\left(b-A_{2} y\right) \leq 0 \quad j=1, \ldots, t \\
& y \in Y .
\end{array}
$$

Introducing variable $\theta,\left(P^{\prime \prime}\right)$ is equivalent to the Benders problem $(B P)$ :

$$
\begin{array}{lll} 
& \min & \theta \\
(B P) \quad \text { s. to } & \theta \geq d^{\top} y+u^{i^{\top}}\left(b-A_{2} y\right) & i=1, \ldots, s \\
& v^{j}\left(b-A_{2} y\right) \leq 0 & j=1, \ldots, t \\
& y \in Y . &
\end{array}
$$

Problem $(B P)$ is impractical since $s$ and $t$ can be very large, and in addition the vertices and extreme rays are unknown. Instead, the method considers a relaxation $\left(B P_{r}\right)$ with a subset of the vertices and extreme rays. The relaxed Benders problem (or master problem) is thus:

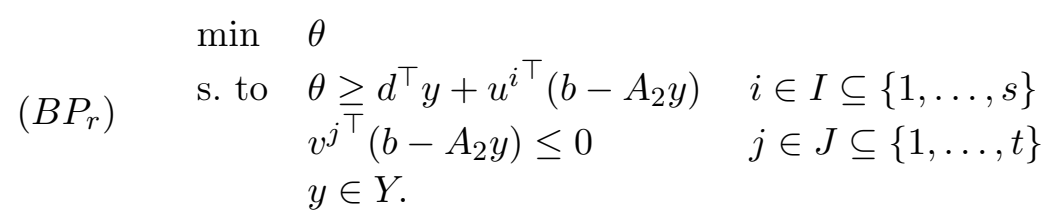

Initially $I=J=\emptyset$, and new vertices and extreme rays provided by the subproblem $\left(Q_{D}\right)$ are added to the master problem, until the optimal solution is found. In summary, the steps of the Benders algorithm are:

\section{Benders algorithm}

0 . Initially $I=\emptyset$ and $J=\emptyset$. Let $\left(\theta_{r}^{*}, y_{r}^{*}\right)$ be the solution of current master problem $\left(B P_{r}\right)$, and $\left(\theta^{*}, y^{*}\right)$ the optimal solution of $(B P)$.

1. Solve master problem $\left(B P_{r}\right)$ obtaining $\theta_{r}^{*}$ and $y_{r}^{*}$. At first iteration, $\theta_{r}^{*}=-\infty$ and $y_{r}$ is any feasible point in $Y$.

2. Solve subproblem $\left(Q_{D}\right)$ using $y=y_{r}^{*}$. There are two cases:

(a) $\left(Q_{D}\right)$ has finite optimal solution in vertex $u^{i 0}$.

- If $\theta_{r}^{*}=d^{\top} y_{r}^{*}+u^{i_{0}}{ }^{\top}\left(b-A_{2} y_{r}^{*}\right)$ then STOP. Optimal solution is $y^{*}=y_{r}^{*}$ with $\operatorname{cost} \theta^{*}=\theta_{r}^{*}$.

- If $\theta_{r}^{*}<d^{\top} y_{r}^{*}+u^{i_{0}}{ }^{\top}\left(b-A_{2} y_{r}^{*}\right)$ then this solution violates constraint of $(B P) \theta>d^{\top} y+u^{i_{0}}{ }^{\top}\left(b-A_{2} y\right)$. Add this new constraint to $\left(B P_{r}\right): I \leftarrow I \cup\left\{i_{0}\right\}$. 
(b) $\left(Q_{D}\right)$ is unbounded along segment $u^{i_{0}}+\lambda v^{j_{0}}\left(u^{i_{0}}\right.$ is current vertex, $v^{j_{0}}$ is extreme ray). Then this solution violates constraint of $(B P)$ $v^{j_{0}}\left(b-A_{2} w\right) \leq 0$. Add this new constraint to $\left(B P_{r}\right): J \leftarrow J \cup\left\{j_{0}\right\}$; vertex may also be added: $I \leftarrow I \cup\left\{i_{0}\right\}$.

3. Go to step 1 above.

Convergence is guaranteed since at each iteration one or two constraints are added to $\left(B P_{r}\right)$, no constraints are repeated, and the maximum number of constraints is $s+t$.

\section{Benders decomposition for the interval protection problem}

Problem (3) has two groups of variables: $x^{l, s} \in \mathbb{R}^{n}, x^{u, s} \in \mathbb{R}^{n}$; and $l b \in$ $\mathbb{R}^{|\mathcal{H}|}, u b \in \mathbb{R}^{|\mathcal{H}|}$, which can be seen as the complicating variables, since if they are fixed, the resulting problem in variables $x^{l, s}$ and $x^{u, s}$ is separable, as shown below. Indeed, projecting out the $x^{l, s}, x^{u, s}$ variables, (3) can be written as

$$
\begin{array}{cl}
\min & \sum_{i \in \mathcal{H}} w_{i}\left(u b_{i}-l b_{i}\right)+Q(u b, l b) \\
\text { s.to } l_{i} \leq l b_{i} \leq a_{i} \quad i \in \mathcal{H} \\
\quad a_{i} \leq u b_{i} \leq u_{i} \quad i \in \mathcal{H}
\end{array}
$$

where

$$
\begin{aligned}
& \begin{array}{c}
Q(u b, l b)=\min _{\text {s.to }}\left(0_{n}^{\top} x^{l, s}+0_{n}^{\top} x^{u, s}\right)=0 \\
A x^{l, s}=b
\end{array} \\
& l_{i} \leq x_{i}^{l, s} \leq u_{i} \quad i \in \mathcal{N} \backslash \mathcal{H} \\
& \begin{aligned}
l b_{i} \leq x_{i}^{l, s} & \leq u b_{i} \quad i \in \mathcal{H} \\
x_{s}^{l, s} & \leq a_{s}-l p l_{s}
\end{aligned} \\
& x_{s}^{l, s} \leq a_{s}-l p l_{s} \quad \forall s \in \mathcal{S}, \\
& \left.\begin{array}{rl}
A x^{u, s} & =b \\
l_{i} \leq x_{i}^{u, s} & \leq u_{i} \quad \quad \quad i \in \mathcal{N} \backslash \mathcal{H} \\
l b_{i} \leq x_{i}^{u, s} & \leq u b_{i} \quad i \in \mathcal{H} \\
x_{s}^{u, s} & \geq a_{s}+u p l_{s}
\end{array}\right\}
\end{aligned}
$$

$0_{n} \in \mathbb{R}^{n}$ denoting the zero vector. Problem (5) is separable in the $x^{l, s}$, $x^{u, s}$ variables for each $s \in \mathcal{S}$ so it can be replaced by the solution of $2|\mathcal{S}|$ 
smaller problems of the form

$$
\begin{array}{rlr}
Q^{l, s}(u b, l b)=\min 0_{n}^{\top} x^{l, s}=0 & & \\
\text { s.to } \quad A x^{l, s} & =b & \\
l_{i} \leq x_{i}^{l, s} & \leq u_{i} \quad & i \in \mathcal{N} \backslash \mathcal{H} \\
l b_{i} \leq x_{i}^{l, s} & \leq u b_{i} \quad & i \in \mathcal{H} \\
x_{s}^{l, s} & \leq a_{s}-l p l_{s},
\end{array}
$$

for the lower protection of sensitive cell $s \in \mathcal{S}$, and

$$
\begin{array}{rlrl}
Q^{u, s}(u b, l b)=\min _{n}^{\top} x^{u, s}=0 & & \\
\text { s.to } \quad A x^{u, s}=b & \\
l_{i} \leq x_{i}^{u, s} \leq u_{i} & i \in \mathcal{N} \backslash \mathcal{H} \\
l b_{i} \leq x_{i}^{u, s} \leq u b_{i} & i \in \mathcal{H}
\end{array}
$$

for the upper protection of sensitive cell $s \in \mathcal{S}$. Note that (5)-(7) are just feasibility problems with a constant (dummy) objective function.

Problems (6) and (7) are our Benders subproblems. Due to its constant objective function, (6) and (7) are feasibility problems. Therefore Benders algorithm will only include extreme rays of the dual formulations of (6) and (7) to guarantee the feasibility of the values of $l b$ and $u b$ provided by the master problem.

Denoting the $j$-th extreme ray of the dual formulation of (6) as $v_{l, s}^{j}=$ $\left(v_{j}^{\lambda^{l, s}}, v_{j}^{\mu_{l}^{l, s}}, v_{j}^{\mu_{u}^{l, s}}, v_{j}^{\nu^{l, s}}\right)$, where $\lambda^{l, s}, \mu_{l}^{l, s}, \mu_{u}^{l, s}$ and $\nu^{l, s}$ refer to the indices of the Lagrange multipliers of the constraints of (6), it can be shown that the feasibility cut to be added to the master problem would be

$$
\begin{aligned}
0 & \geq \sum_{i=1}^{m} v_{j, i}^{\lambda^{l, s}} b_{i}+\sum_{i \in \mathcal{N} \backslash \mathcal{H}}\left(-v_{j, i}^{\mu_{u}^{l, s}} u_{i}+v_{j, i}^{\mu_{l}^{l, s}} l_{i}\right)+\sum_{i \in \mathcal{H}}\left(-v_{j, i}^{\mu_{u}^{l, s}} u b_{i}+v_{j, i}^{\mu_{l}^{l, s}} l b_{i}\right)-\left(a_{s}-l p l_{s}\right) v_{j}^{\nu^{l, s}} \\
& =g_{l, s}^{j}(u b, l b) .
\end{aligned}
$$

The extreme rays of the dual of (7) have an analogous form $v_{u, s}^{j}=$ $\left(v_{j}^{\lambda^{u, s}}, v_{j}^{\mu_{l}^{u, s}}, v_{j}^{\mu_{u}^{u, s}}, v_{j}^{\nu^{u, s}}\right)$ and so does the feasibility cut to be added to the master problem:

$$
\begin{aligned}
0 & \geq \sum_{i=1}^{m} v_{j, i}^{\lambda^{u, s}} b_{i}+\sum_{i \in \mathcal{N} \backslash \mathcal{H}}\left(-v_{j, i}^{\mu_{u}^{u, s}} u_{i}+v_{j, i}^{\mu_{l}^{u, s}} l_{i}\right)+\sum_{i \in \mathcal{H}}\left(-v_{j, i}^{\mu_{u}^{u, s}} u b_{i}+v_{j, i}^{\mu_{l}^{u, s}} l b_{i}\right)+\left(a_{s}-l p l_{s}\right) v_{j}^{\nu^{u, s}} \\
& =g_{u, s}^{j}(u b, l b) .
\end{aligned}
$$


Denoting as $\mathcal{I}_{l, s}$ and $\mathcal{I}_{u, s}$ the set of indices of feasibility cuts obtained from $Q^{l, s}$ and $Q^{u, s}$, the master problem is:

$$
\begin{array}{cl}
\min \sum_{i \in \mathcal{H}} w_{i}\left(u b_{i}-l b_{i}\right) & \\
\text { s.to } g_{j}^{l, s}(u b, l b) \leq 0 & j \in \mathcal{I}_{l, s} \\
g_{j}^{u, s}(u b, l b) \leq 0 & j \in \mathcal{I}_{u, s} \\
l_{i} \leq l b_{i} \leq a_{i} & i \in \mathcal{H} \\
a_{i} \leq u b_{i} \leq u_{i} & i \in \mathcal{H} .
\end{array}
$$

The Benders decomposition algorithm will then solve (10) for the master problem and the duals of (6) and (7) for the subproblems.

\subsection{Illustrative example}

Consider the following simple table

$$
\begin{array}{|ll|l|}
\hline 10 & 15 & 25 \\
20 & 17 & 37 \\
\hline
\end{array}
$$

of $n=6$ cells and $m=2$ linear constraints associated to row totals

$$
\begin{aligned}
& a_{1}+a_{2}-a_{3}=0 \\
& a_{4}+a_{5}-a_{6}=0
\end{aligned}
$$

(we don't consider column totals to simplify the example), where $\mathcal{H}=$ $\mathcal{N}=\{1, \ldots, 6\}$, and $a_{1}$ and $a_{5}$ as the two sensitive cells, whose parameters are given by

\begin{tabular}{rrrr}
\hline$s$ & $a_{s}$ & $l_{p} l_{s}$ & $u p l_{s}$ \\
\hline 1 & 10 & 5 & 5 \\
5 & 17 & 7 & 4 \\
\hline
\end{tabular}

Note that this example, in principle, can not be solved with the original implementation of [10] since the ratios between upper and lower protection levels are not the same for all sensitive cells.

We next show the application of Benders algorithm to the previous table:

\section{Initialization}

The number of cuts for the $l b$ and the $u b$ variables is set to 0 , this means $\mathcal{I}_{l, s}=\mathcal{I}_{u, s}=\emptyset$. The first master problem to be solved is thus

$$
\begin{aligned}
& \min \sum_{i=1}^{6}\left(u b_{i}-l b_{i}\right) \\
& \text { s.to } l_{i} \leq l b_{i} \leq a_{i} \quad i=1, \ldots, 6 \\
& a_{i} \leq u b_{i} \leq u_{i} \quad i=1, \ldots, 6,
\end{aligned}
$$

obtaining some initial values for $l b, u b$. 


\section{Iterating through Benders' algorithm}

Cut generation is based on (8)-(9), details are omitted to simplify the exposition.

- Iteration 1. The two Benders cuts obtained for cell 1 are $l b_{1} \leq$ 5 and $u b_{1} \geq 15$. The two Benders cuts obtained for cell 5 are $l b_{5} \leq 10$ and $u b_{1} \geq 21$. Note these are obvious cuts associated to the protection levels of sensitive cells, that could have been added from the beginning in an efficient implementation, thus avoiding this first Benders iteration.

- Iteration 2. The current master subproblem

$$
\begin{aligned}
& \min \sum_{i=1}^{6}\left(u b_{i}-l b_{i}\right) \\
& \text { s.to } l_{i} \leq l b_{i} \leq a_{i} \quad i \in 1, \ldots, 6 \\
& a_{i} \leq u b_{i} \leq u_{i} \quad i \in 1, \ldots, 6 \\
& l b_{1} \leq 5, \quad u b_{1} \geq 15 \\
& l b_{5} \leq 10, \quad u b_{5} \geq 21
\end{aligned}
$$

has solution $l b=[5,15,25,20,10,37]$ and $u b=[15,15,25,20,21,37]$. Using this solution the two Benders cuts obtained for cell 1 are $l b_{3}-u b_{2} \leq 58$ and $l b_{2}-u b_{2} \geq 15$. The two cuts obtained for cell 5 are $l b_{6}-u b_{4} \leq 21$ and $u b_{6}-l b_{4} \geq 39$.

- Iteration 3. The current master problem is

$$
\begin{aligned}
& \min \sum_{i=1}^{6}\left(u b_{i}-l b_{i}\right) \\
& \text { s.to } l_{i} \leq l b_{i} \leq a_{i} \quad i \in 1, \ldots, 6 \\
& a_{i} \leq u b_{i} \leq u_{i} \quad i \in 1, \ldots, 6 \\
& l b_{1} \leq 5, \quad u b_{1} \geq 15 \\
& l b_{5} \leq 10, \quad u b_{5} \geq 21 \\
& l b_{3}-u b_{2} \leq 58, l b_{2}-u b_{2} \geq 15 \\
& l b_{6}-u b_{4} \leq 21, u b_{6}-l b_{4} \geq 39 \text {, }
\end{aligned}
$$

with solution $l b=[5,15,20,16,10,30]$ and $u b=[15,15,30,20,21,37]$. Benders subproblems happen to be feasible with these values, thus we have an optimal solution of objective $\sum_{i=1}^{6}\left(u b_{i}-l b_{i}\right)=42$. Since this table is small, the original model was solved using some off-the-shelf optimization solver, obtaining the same optimal objective function.

3. Auditing. Although this step is not needed with interval protection, to be sure that this solution satisfies that no attacker can determine that $a_{s} \in\left(a_{s}-l p l_{s}, a_{s}+u p l_{s}\right)$ for $s \in\{1,5\}$, the problems (2) were solved, obtaining $\underline{a_{1}}=5, \overline{a_{1}}=15, \underline{a_{5}}=10$ and $\overline{a_{5}}=21$. Therefore, it can be asserted that it is safe to publish this solution. 
4. Publication of the table. The final safe table to be published would be

\begin{tabular}{|rr|r|}
{$[5,15]$} & 15 \\
{$[16,20]$} & {$[10,21]$} & {$[30,30]$} \\
{$[30,37]$}
\end{tabular}.

\section{Computational results}

We developed a prototype implementation of the Benders algorithm for interval protection using the AMPL modeling language [11] and Cplex for the master and subproblems. We solved seven instances, whose dimensions are given in Table 1. Columns $n,|\mathcal{S}|$ and $m$ provide, respectively, the number of cells, sensitive cells and table linear equations. Table "targus" is a general table, while the remaining six tables are $1 \mathrm{H} 2 \mathrm{D}$ tables (i.e., twodimensional hierarchical tables with one hierarchical variable) obtained with a generator used in the literature [18].

Table 1. Instance dimensions and results with Benders decomposition

\begin{tabular}{lrrrrrrr}
\hline table & $n$ & $|\mathcal{S}|$ & $m$ & $\mathrm{CPU}$ & it $_{B}$ & it $_{S}$ & obj \\
\hline targus & 162 & 13 & 63 & 5.17 & 31 & 8872 & 2142265.7 \\
table1 & 121 & 10 & 55 & 3.41 & 26 & 7167 & 136924 \\
table2 & 1680 & 158 & 299 & 410.53 & 43 & 1104884 & 43715149 \\
table3 & 600 & 53 & 170 & 26.38 & 43 & 131834 & 3624906 \\
table4 & 756 & 68 & 243 & 50.92 & 33 & 144963 & 9134139 \\
table5 & 168 & 14 & 62 & 3.95 & 19 & 5959 & 303844 \\
table6 & 1584 & 143 & 485 & 966.28 & 70 & 1729767 & 21302104 \\
\hline
\end{tabular}

Table 2. Results using Cplex for monolithic model

\begin{tabular}{lrrrr}
\hline table & CPU & it $_{S}$ & n.var & obj \\
\hline targus & 36.0515 & 16532 & 4212 & 2142265.7 \\
table1 & 3.43548 & 7452 & 2420 & 136924 \\
table2 & ${ }^{*} 2944.87$ & - & 530880 & 16056608400 \\
table3 & ${ }^{*} 522.875$ & - & 63600 & 260592812 \\
table4 & 11085.6 & 436895 & 102816 & 9134139 \\
table5 & 10.6764 & 17325 & 4704 & 303844 \\
table6 & $* 7816.61$ & - & 453024 & 4404161015 \\
\hline * Aborted due to excessive CPU time
\end{tabular}


The results obtained with the Benders decomposition are provided in the last columns of Table1. Columns "CPU", "it ${ }_{B}$ ", "it $S$ " and "obj" provide respectively the total CPU time, number of Benders iterations, overall number of simplex iterations, and the final optimal objective function obtained.

Table 2 provides results for the solution of the monolithic model (3) using Cplex default linear algorithm (dual simplex). Column "n.var" reports the number of variables of the resulting linear optimization problem. The meaning of remaining columns is the same as in Table1. Three executions, clearly marked, were aborted because the CPU time was excessive compared with the solution by Benders; in those cases column "obj" provides the value of the objective function when the algorithm was stopped. From these tables it is clear that the solution of the monolithic model is impractical and that an standard implementation of Benders can be more efficient for some classes of problems (namely, 1H2D tables).

\section{Conclusions}

Partial cell suppression or interval protection can be an alternative method for tabular data protection. Unlike other approaches, this method results in a huge but continuous optimization problem, which can be effectively solved by linear optimization algorithms. One of them is Benders decomposition: a prototype code was able to solve some nontrivial tables more efficiently than state-of-the-art solvers applied to the monolithic model. It is expected that a more sophisticated implementation of Benders algorithm would be able to solve even larger and more complex tables. An additional and promising line of research would be to consider highly efficient specialized interior-point methods for block-angular problems [76]. This is part of the further work to be done.

\section{References}

1. Baena, D., Castro, J., González, J.A.: Fix-and-relax approaches for controlled tabular adjustment. Computers \& Operations Research 58 (2015) 41-52

2. Benders, J.F.: Partitioning procedures for solving mixed-variables programming problems. Computational Management Science 2 (2005) 3-19. English translation of the original paper appeared in Numerische Mathematik, 4 (1962) 238-252

3. Castro, J.: Minimum-distance controlled perturbation methods for large-scale tabular data protection. European Journal of Operational Research 171 (2006) 39-52

4. Castro, J.: A shortest paths heuristic for statistical disclosure control in positive tables. INFORMS Journal on Computing 19 (2007) 520-533. 
5. Castro, J.: Recent advances in optimization techniques for statistical tabular data protection. European Journal of Operational Research 216 (2012) 257-269

6. Castro, J.: Interior-point solver for convex separable block-angular problems. Optimization Methods \& Software 31 (2016) 88-109

7. Castro, J., Cuesta, J.: Quadratic regularizations in an interior-point method for primal block-angular problems. Mathematical Programming 130 (2011) 415-445

8. Castro, J., Frangioni, A., Gentile, C.: Perspective reformulations of the CTA problem with $L_{2}$ distances. Operations Research 62 (2014) 891-909

9. Fischetti, M., Salazar, J.J.: Solving the cell suppression problem on tabular data with linear constraints. Management Science 47 (2001) 1008-1026

10. Fischetti, M., Salazar, J.J.: Partial cell suppression: A new methodology for statistical disclosure control. Statistics and Computing 13 (2003) 13-21

11. Fourer, R., Gay, D.M., Kernighan, D.W.: AMPL: A Modeling Language for Mathematical Programming. Duxbury Press (2002)

12. Hundepool, A., Domingo-Ferrer, J., Franconi, L., Giessing, S., Schulte-Nordholt, E. Spicer, K., de Wolf, P.P.: Statistical Disclosure Control, Wiley, Chichester (2012)

13. Wright, S.J.: Primal-Dual Interior-Point Methods. SIAM, Philadelphia (1997) 